\title{
An Indoor Contaminant Sensor Placement Toolbox for Critical Infrastructure Buildings
}

\author{
Demetrios G. Eliades ${ }^{1 \star}$, Michalis P. Michaelides ${ }^{1,2}$, Marinos Christodoulou ${ }^{1}$, \\ Marios Kyriakou $^{1}$, Christos G. Panayiotou ${ }^{1}$, and Marios M. Polycarpou ${ }^{1}$ \\ 1 KIOS Research Center for Intelligent Systems and Networks, and \\ Department of Electrical and Computer Engineering \\ University of Cyprus \\ 75 Kallipoleos Ave., CY-1678 Nicosia,Cyprus \\ 2 Department of Electrical Engineering and Information Technologies \\ Cyprus University of Technology \\ 30 Archbishop Kyprianos Str., CY-3036 Lemesos, Cyprus
}

\begin{abstract}
In this work, we address the problem of airborne contaminant sensor placement in high-risk buildings where critical infrastructures are managed and operated, making them possible locations for terrorist attacks (such as governmental buildings and ministries, utilities, airports and hospitals). A new software is presented based on the "Matlab-CONTAM Toolbox" and the CONTAM multi-zone simulation software, to construct multiple scenarios of contamination events and to solve the multi-objective sensor placement problem for minimizing the average and maximum impact risk with respect to the contaminant mass inhaled impact metric. The use of the software is demonstrated in a case-study using the Holmes's House benchmark. The Toolbox is released under an open-source license at https://github.com/KIOS-Research/ matlab-contam-toolbox.
\end{abstract}

Keywords: Sensor Placement, High-Risk Buildings, Critical Infrastructure Protection, Contamination, MATLAB-CONTAM Toolbox

\section{Introduction}

According to the European Council Directive 2008/114/EC, Critical Infrastructures are defined as those systems which are essential for maintaining the societal and economic well-being of the people, and in case their operation would be disrupted or destroyed, the state would fail in maintaining those functions [8]. Infrastructures such as electricity, oil, gas, transportation, water and telecommunication systems are considered critical; this includes both cyber and physical layers as well as their associated facilities $[7,8]$. Critical infrastructures are susceptible to various types of faults which may be due to environmental conditions, human errors or malicious attacks. For this reason, Critical Infrastructure Protection has received significant interest by governments and researchers in the

\footnotetext{
* Corresponding author: eldemet@ucy.ac.cy.
} 
last decade, in order to identify risks leading to failure, study interdependencies between infrastructures, design tools for early detection of faults and software models, as well as protocols and controls for mitigating attacks [10].

In this work, we investigate the protection of buildings, which may be the operational or management centers of critical infrastructures, such as governmental buildings and ministries, utilities, airports and hospitals. In case of an attack in a critical infrastructure building, the overall operation of the corresponding infrastructure could be affected. A type of terrorist attack in buildings is through the release of some airborne chemical, biological or radiological agent, and such an event could affect the health and safety of the occupants in the different building zones dramatically [11]. In case a dangerous substance is released within a building zone, the substance would spread through the different parts of the building as a result of the air-flows between the different zones. Sensor information can be utilized to alert the occupants as well as the operators to take the appropriate measures in the case of a contaminant release. Ideally, it would be desirable to have sensors in every room of the building, measuring all different types of contaminants, but the cost and sophistication of most contaminant sensors today prohibits this. In practice, a small number of sensors will be available, with respect to the number of building zones which need to be covered. When solving this problem one should take into account the topology of the building, the significance of each zone as well as to consider uncertainties in the parameters considered. Determining where to install a limited number of sensors for contaminant detection is a non-trivial problem, as multiple and conflicting objectives need to be satisfied, such as coverage, detection time, number of people affected, installation costs etc.

The problem of selecting optimal sensor locations has also received significant interest in various fields, such as operational research [15], control systems [2] and water distribution systems security [13,6]. In [1], the optimal sensor locations were determined for detecting releases in a building by using a Computational Fluid Dynamics (CFD) tool to estimate the distribution of contaminants. In [19], CFD techniques were also applied to predict chemical and biological agent dispersion in an office complex for finding the best locations for sensors and for developing effective ventilation strategies. Multi-zone models have also been used, for instance, in [4], six attack scenarios for a small commercial building were simulated, and a genetic algorithm was applied for each attack scenario to optimize the sensor sensitivity, location, and number to achieve the best system behavior while minimizing system cost. In [3], the impact of zonal and multi-zone modeling techniques on indoor air protection systems was analyzed for a typical office environment and a large hall. The use of a systems engineering approach utilizing contamination attack scenarios, criteria for evaluating performance as well as the use of contaminant sensor technologies, has been advocated by [9]. Under these safety-critical situations, it becomes of paramount importance that the contaminant is promptly detected and localized so that appropriate control actions are taken to mitigate the damage and ensure the safety of the people [12]. A software tool for "smart buildings" was presented by [14], as a decision 
support tool for determining where to install contamination sensors, in order to reduce detection time. Probability distributions are considered for the different parameters, such as the release zone, the chemical agent quantity and the release duration.

The focus of this work is to present a new decision support tool for making recommendations regarding where to install a number of sensors for airborne contaminants. Compared to previous works, our approach differentiates in that it constructs multiple environmental and contamination scenarios based on sampling of the different probability distributions, taking into account building utilization and people distribution, for minimizing multiple impact-risk objectives. In our previous work [5], a methodology was presented for determining where to install a small number of contaminant sensors. First, a set of contamination scenarios is constructed, and each of the different scenarios is simulated using a multi-zone building simulation software. For assessing the damage caused by each scenario (e.g., number of people infected) we calculate an impact metric based on the total amount of contaminant inhaled and depending on the number of people, their age and type of their activity within the building. Finally, for deciding on where to place the sensors we solve an optimization problem that may involve multiple objectives, for instance to minimize (i) the average impact damage, (ii) the worst-case impact damage and (iii) the cost/number of sensors. In general, compared to existing simpler methods, this method allows better handling of more complex scenarios which involve many parameters and different optimization functions, as well as taking into account the building usage [5].

CONTAM [17] is a multi-zone simulation software developed by the US National Institute of Standards and Technology (NIST), with which the user can easily create the building outline and specify the zone volumes, the openings, the environmental conditions, as well as the contaminant sources present, to compute airflows and simulate contaminant concentrations in the different building zones. The computational engine of CONTAM has been used in various building security studies to simulate contaminant propagation $[9,12,5]$.

Some of the key challenges in security research is to compare different methods through benchmarks as well as to implement usable software solutions so that these algorithms can be evaluated by the industry. This motivates the work presented in this paper, of a new software implemented in Matlab for computing sensor placement solutions based on the mathematical framework proposed in [5]. The software is based on the "Matlab-CONTAM Toolbox", a new development platform for researchers and the industry, which integrates a building simulation tool (CONTAM) as well as scenario construction and optimization algorithms, in order to be used as a benchmarking tool among the critical infrastructure protection community. Through the Toolbox, a large number of contamination scenarios under different conditions can be simulated, and these data can be used for further analysis by the different algorithms. In specific, the Toolbox allows the creation of multiple contamination event scenarios by varying the different problem parameters (wind direction, wind speed, leakage path 
openings, source magnitude, evolution rate and onset time). The use of the Toolbox is illustrated through the use of a case study based on the Holmes's House benchmark [18]. The software is released under an open-source license and is available at https://github.com/KIOS-Research/matlab-contam-toolbox.

The remaining of this paper is organized as follows: In Section 2, the mathematical problem formulation is described as used in the developed software. In Section 3, the modular software architecture is presented. In Section 4, a case study is presented to illustrate the usage of the Toolbox and the Sensor Placement methodology. Finally, Section 5 concludes the paper and future work is discussed.

\section{Problem Formulation}

The indoor contaminant dispersion dynamics model and the sensor placement methodology, as utilized in the software, are described in this section. The goal is to solve a combinatorial problem for deciding where to install contamination sensors in a large high-risk building, in order to reduce the possibility of severe damage due to an airborne contaminant event which may affect the critical infrastructure operation.

In the following, the formulation used within the software is presented. Further details on the multi-zone model can be found in [12], along with the relation of the different model components and the mass-balance equations. The model used can represent both naturally and mechanically ventilated buildings. In addition, sources and sink elements can be incorporated in the model. Additional details in relation to the sensor placement methodology can be found in [5].

Let $\mathcal{R}$ represent the set of real numbers. The state-space equations for contaminant dispersion in an indoor building environment with $N$ zones, as considered in this software, are described by

$$
\dot{x}=A\left(p_{x}\right) x+\phi\left(p_{x}, p_{\phi}\right) .
$$

The vector $x \in \mathcal{R}^{N}$ represents the concentration of the contaminant in the building zones (measured in mass per volume). The state matrix $A \in \mathcal{R}^{N \times N}$ models the changes in the contaminant concentrations between the different building zones as a result of the airflows and is a function of vector $p_{x}$, which is comprised of the wind-direction and wind-speed, the ambient temperature, the volumes of all zones, the temperatures in all zones and the opening status of all doors, windows and fans. The term $\phi \in \mathcal{R}^{N}$ represents the contaminant mass release into the building due to an attack, and $p_{\phi}$ is a vector comprised of the locations where contaminants are released, their release duration, as well as their release rates.

To measure the damage caused during a contamination scenario at the $k$-th zone, an impact value $z_{k}$ can be computed. This corresponds to the damage caused on the system measured through some impact metric. In this work, the contaminant mass inhaled is considered, which is given by

$$
\dot{z}_{k}=f_{z}\left(x_{k} ; p_{z}\right)
$$


where $f_{z}(\cdot)$ is the function for computing the change rate of the impact $z_{k}$ for an airborne contamination event at the $k$-th zone. This depends on the contaminant concentration $x_{k}$ and the vector $p_{z}$, which is comprised of the average daily zone occupancy (proportional to the actual usage of some zone by people), the inhalation rate (which depends on the building/zone functionality), and the concentration threshold of the sensors.

In general, the parameter vectors $p_{x}$ and $p_{\phi}$ may be partially/nominally known, and these uncertainties may influence the calculations of the sensor placement solutions. In this work, multiple scenarios with different parameter values are considered, to better capture the differences between the actual system and the model. Uniform bounds of each parameter are considered known, and the user can decide the number of samples within the range of each parameter.

A finite set $\mathcal{P}$ is constructed and is comprised of $N_{p}$ elements corresponding to all the combinations of the different parameter sampled values considered. For each contamination scenario in $\mathcal{P}$, the indoor contaminant dispersion dynamics are simulated for a period of $T$ hours. Let $\Omega$ be the overall-impact matrix

$$
\Omega=\left[\begin{array}{ccc}
\Omega_{1,1} & \cdots & \Omega_{1, N} \\
\vdots & \ddots & \vdots \\
\Omega_{N_{p}, 1} & \cdots & \Omega_{N_{p}, N}
\end{array}\right]
$$

such that its $(i, j)$-th element, $\Omega_{i, j}$ corresponds to the total contaminant mass inhaled due to the $i$-th contamination scenario from $\mathcal{P}$, assuming a sensor is monitoring the $j$-th zone.

Finally, the optimization problem for contaminant sensor placement in highrisk buildings is formulated as a multi-objective risk-minimization problem, where the best solutions belong to a Pareto Front with respect to certain objectives. Specifically, in this software, the multi-objective optimization problem is formulated in the software as

$$
Y=\underset{\chi \in\{1,0\}^{N}}{\operatorname{argmin}}\left\{F_{0}(\chi), F_{1}(\chi ; \Omega), F_{2}(\chi ; \Omega)\right\},
$$

where $\chi$ is the zone index set for which $\chi_{l}=1$ when a sensor is installed and $\chi_{l}=0$ when there is no sensor installed at the $l$-th zone. Function $F_{0}$ corresponds to the number of sensors which depends on the user input, $F_{1}$ is the estimated average impact-risk and $F_{2}$ is the estimated worst-case impact-risk. For computing the best Pareto Front solutions, the selection of the algorithm depends on the problem size. For small problems, an exhaustive search may be computationally feasible, whereas for larger problem other methods such as multi-objective evolutionary optimization algorithms can be applied.

After solving the optimization problem and the Pareto Solutions set has been constructed, decision makers may use higher level reasoning to arrive at the final decision regarding which zones to install the contamination sensors. 


\section{Software Architecture}

The Matlab-CONTAM Toolbox provides a programming interface for CONTAM, a multizone airflow and contaminant transport analysis software. The goal of the Toolbox is to serve research and industry by facilitating the simulation of multiple contamination events under varying conditions as well as to store computed results in data structures so that they can be reused by different algorithms. An intuitive Graphical User Interface (GUI) has been designed to access the different functionalities of the Toolbox and the Sensor Placement algorithm. It is important to note that the architecture of the software allows for new modules to be added to the Toolbox. The Software Architecture is depicted in Fig. 1.

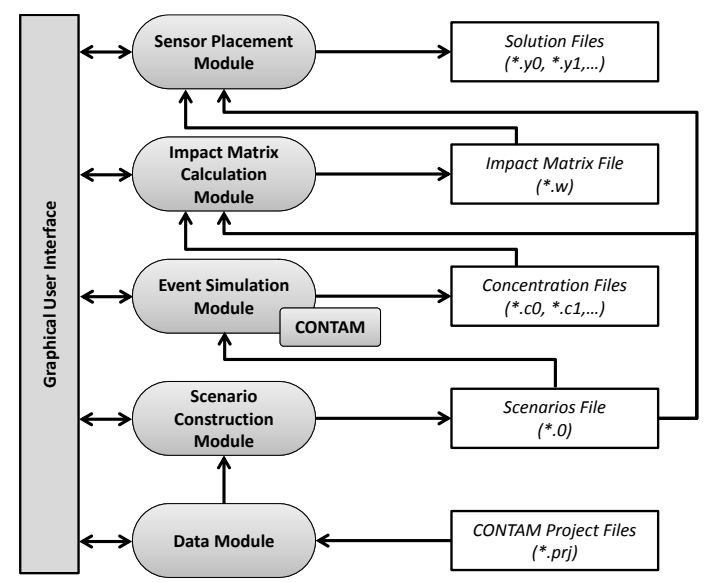

Fig. 1. The software architecture of the Matlab-CONTAM Toolbox and the Sensor Placement module.

The software acquires the building information from CONTAM Project files, which are comprised of details regarding walls, floors, ceilings, air-handling systems, ducts, weather conditions etc. The "Data Module" opens CONTAM Project files (*.prj) and extracts the information which is related to the building parameters. In addition, the building zone schematics are extracted and this information is used by the GUI of the Toolbox to plot the building schematics.

The information from the "Data Module" is then used by the "Scenario Construction Module". This module assists for specifying, through the GUI, the building parameters for constructing a single or multiple simulation scenarios. The different contamination scenario parameters, along with structural and environmental information are stored in the Scenarios File (*.0).

The data stored in the Scenarios File is used by the "Event Simulation Module", which is responsible for communicating with the CONTAM engine to compute the path air-flows for different environmental conditions. When the flows 
have been computed, the module simulates the different contamination scenarios and computes the contaminant concentration at each building zone. The contaminant concentrations are then stored in one or more Concentration Files $(* . \mathrm{c} 0, * . \mathrm{c} 1, \ldots)$.

The "Impact Matrix Calculation Module" utilizes the data from the scenarios and the simulation files, as well as data from the GUI (such as the average daily zone occupancy), to calculate the damage caused by some contamination event, until the time the contaminant exceeds some concentration at a certain zone. The matrix computed for all the possible scenarios and all the possible zones is stored in the Impact Matrix File (*.w).

Finally, the "Sensor Placement Module" is used to compute the final solutions, based on the computed impact matrices and the scenarios file. Through the GUI, the user specifies which method to use to solve the problem. For instance, exhaustive search methods would compute all the possible solution combinations, and calculate the Pareto Solutions. The solutions can be depicted graphically on the Interface, and are stored in a Solutions File (e.g. * .y0 for the exhaustive search method, *.y1 for the evolutionary computation method)

\section{Case Study}

In the following we present a case study for the high-risk building sensor placement software using a realistic benchmark based on the Holmes's House [18] which is depicted in Fig. 2. The building is comprised of 14 zones: a garage (Z1), a storage room (Z2), a utility room (Z3), a living room (Z4), a kitchen (Z5), two bathrooms (Z6 and Z13), a corridor (Z8), three bedrooms (Z7, Z9 and Z14) and three closets (Z10, Z11 and Z12). There are 30 path openings corresponding to windows and doors (P1-P30).

The first part of the sensor placement algorithm is the construction and simulation of multiple scenarios describing different environmental, structural and attack conditions. Figure 3 depicts the interface for selecting the scenario parameters: the simulation time and the time step for solving numerically the differential equations, the maximum number of simultaneous contamination sources, as well as the contaminant parameters and the environmental/structural parameters which affect flows. Where available, the building's nominal values are loaded (e.g. zone volumes, wind direction and speed, ambient and zone temperatures, path openings). However, the user can modify any of these parameters as required. The user can specify upper and lower bounds for each parameter using a percentage, for instance, for a wind speed $10 \mathrm{~m} / \mathrm{s}$, a $10 \%$ bound corresponds to a wind velocity within the range $[9,11] \mathrm{m} / \mathrm{s}$. Furthermore, the user can select how many samples to segment this range of values, for example, by taking 3 samples from the wind speed range $[9,11] \mathrm{m} / \mathrm{s}$, the set of wind speeds is $\{9,10,11\} \mathrm{m} / \mathrm{s}$. In this work, grid-based parameter selection is considered.

In the following step, all the contamination scenarios are simulated in order to compute the time-series describing the contaminant concentrations in each zone, through the interface depicted in Fig. 4. The path air-flows are computed using 
Fig. 2. The Matlab-CONTAM Toolbox with the Holmes's House project opened.

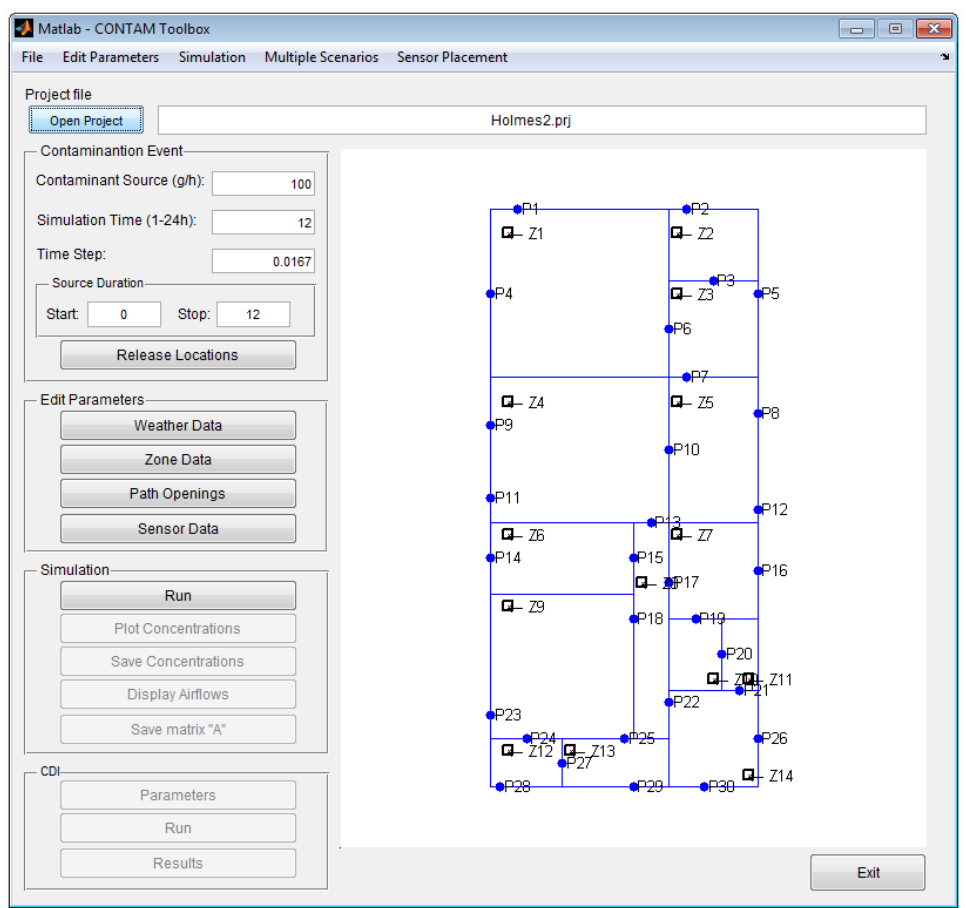

Fig. 3. The interface for selecting scenario parameters and constructing the scenarios set.

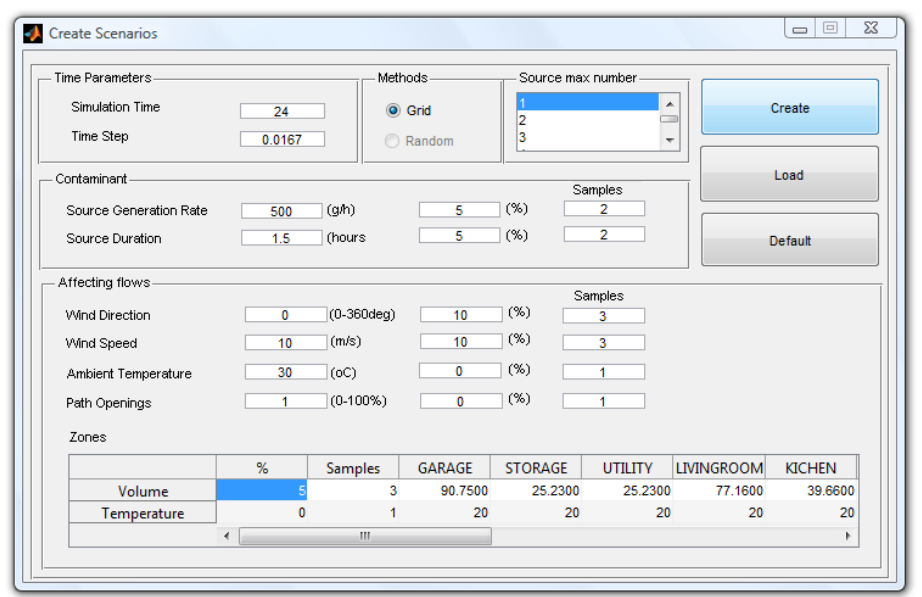


CONTAM, and these flows are used to construct matrix $A\left(p_{x}\right)$ which is used in (1) to solve the equation for the different contamination scenario parameters.

Fig. 4. The interface for running multiple scenarios.

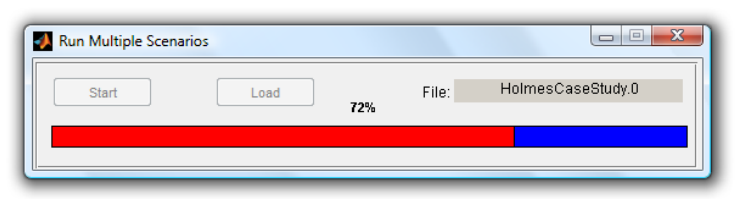

By using the computed contaminant concentration time-series for each zone, the impact matrix $\Omega$ can be constructed for solving the optimization problem. The $(i, j)$-th element of the impact matrix $\Omega$ corresponds to the damage caused due to the $i$-th contamination scenario with respect to having a sensor installed in the $j$-th zone. In this work, the contaminant mass inhaled metric is considered as a metric of the impact damage (2), however different metrics could be implemented as well. The interface for constructing the impact matrix is show in Fig. 5. For each zone, the user can specify an occupancy value, which corresponds to the average number of people residing within a certain zone within a day. For instance, if 10 people reside within a zone for 6 hours each day, the average daily occupancy of that zone would be $10(6 / 24)=2.5$. The inhalation rate depends on the type of work which can be estimated using guidelines [16]. The sensor threshold corresponds to the concentration above which a certain sensor can detect the contaminant, according to its specifications.

Fig. 5. The interface for constructing the impact matrix.

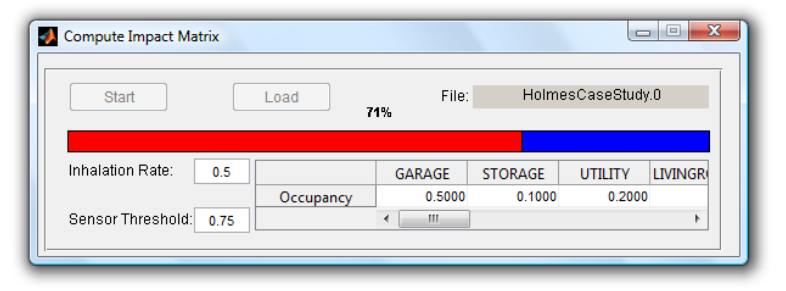

The final step is to solve the optimization problem for sensor placement, using the interface depicted in Fig. 6. The user can select between two methods for constructing the solutions to evaluate the Pareto Front, using the Matlab's multiobjective evolutionary-based algorithm (gamultiobj) which is suitable for larger 
problems, and an exhaustive method which is suitable for smaller problems. The user can select different number of sensors to solve the optimization. The Pareto Solutions are computed for each different number of sensors, the final solutions are shown to the user as in Fig. 7. Through the graphic interface, the user can select different solutions and examine visually the suggested locations for installing sensors, indicated with red dots.

In this example, for the 4-sensor placement problem, there are two Pareto optimal solutions: both solutions suggest installing sensors in the living-room and the two bedrooms, however the solution with the lowest mean risk impact suggest to install the last sensor in a closet, and the solution with the lowest maximum risk impact suggest to install the last sensor in the bath. It is important to note that even though both solutions are Pareto optimal, i.e. one does not dominates the other, the second solution has a significantly lower maximum risk impact with only a moderate increase in the average risk impact objective, and thus a decision maker might choose the second rather than the first solution.

Fig. 6. The interface for solving the sensor placement problem.

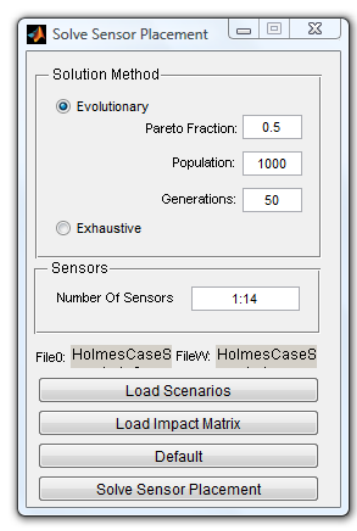

\section{Conclusions and Future Work}

In this work, we examine the problem of improving security in high-risk critical infrastructure buildings from malicious airborne contaminations, by determining where to install a limited number of sensors, while taking into account the building usage and the uncertainty in the structural and environmental parameters. A new decision support software implemented in Matlab for computing sensor placement solutions is presented based on the mathematical framework proposed in [5] and the "Matlab-CONTAM Toolbox", a development platform for 
Fig. 7. The Sensor Placement solutions interface.

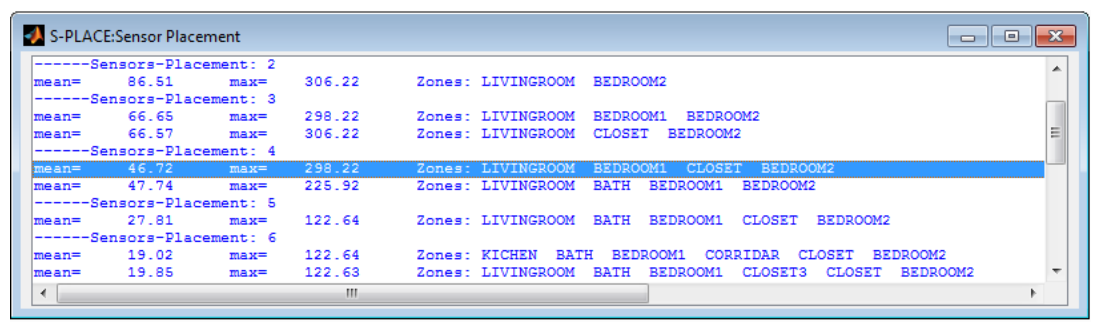

researchers and the industry, which integrates the building simulation software CONTAM as well as other scenario construction and optimization algorithms.

The software has been designed to be used both by the professional as well as by the academic community, making it easy to evaluate solutions under various scenarios, through an intuitive Graphical User Interface. The modular software architecture allows each part of the software data and algorithms to be accessed independently. New methods can be added to investigate the use of different metrics and optimization algorithms, or to solve new problems related with highrisk building security. In this paper, the use of the software is illustrated through the use of a case study based on the Holmes's House benchmark [18]. The Toolbox is released under an open-source license, and can be downloaded at https: //github.com/KIOS-Research/matlab-contam-toolbox.

Future versions of the software will allow selecting different and more sophisticated methods for constructing the scenario parameters, allow the use of different impact metrics and response time-delays to construct multiple impact metrics as well as to allow different optimization methods for constructing the Pareto Solutions.

Acknowledgements This research work has been funded by the European Research Council (ERC) under the project ERC-2011-ADG-291508 "Fault-Adaptive Monitoring and Control of Complex Distributed Dynamical Systems"(FAULTADAPTIVE).

\section{References}

1. Arvelo, J., Brandt, A., Roger, R., Saksena, A.: An enhanced multizone model and its application to optimum placement of CBW sensors. ASHRAE Transactions 108(2), 818-826 (2002)

2. Basseville, M., Benveniste, A., Moustakides, G., Rougee, A.: Optimal sensor location for detecting changes in dynamical behavior. IEEE Transactions on Automatic Control 32(12), 1067 - 1075 (Dec 1987)

3. Chen, Y., Wen, J.: Application of zonal model on indoor air sensor network design. In: Proc. of SPIE (2007) 
4. Chen, Y., Wen, J.: Sensor system design for building indoor air protection. Building and Environment 43(7), 1278-1285 (Jul 2008)

5. Eliades, D., Michaelides, M., Panayiotou, C., Polycarpou, M.: Security-oriented sensor placement in intelligent buildings. Building and Environment 63(0), 114 $121(2013)$

6. Eliades, D., Polycarpou, M.: A fault diagnosis and security framework for water systems. IEEE Transactions on Control Systems Technology 18(6), $1254-1265$ (Nov 2010)

7. European Commission: Critical infrastructure protection in the fight against terrorism. Communication (Oct 2004), COM/2004/0702

8. European Commission: Council directive 2008/114/ec of 8 december 2008 on the identification and designation of european critical infrastructures and the assessment of the need to improve their protection. Official Journal of the European Union L345(23), 12 (2008)

9. Ko, H.W.: Countermeasures against chemical/biological attacks in the built environment. Johns Hopkins APL technical digest 24(4), 360-367 (2003)

10. Lopez, J., Setola, R., Wolthusen, S.D.: Overview of critical information infrastructure protection. In: Lopez, J., Setola, R., Wolthusen, S. (eds.) Critical Infrastructure Protection, Lecture Notes in Computer Science, vol. 7130, pp. 1-14. Springer Berlin Heidelberg (2012)

11. Mead, K.R., Gressel, M.G.: Protecting building environments from airborne chemical, biological, or radiological attacks. Applied Occupational and Environmental Hygiene 17(10), 649-658 (2002), pMID: 12363204

12. Michaelides, M., Reppa, V., Panayiotou, C., Polycarpou, M.: Contaminant event monitoring in intelligent buildings using a multi-zone formulation. In: Proc. of SAFEPROCESS. Mexico City, Mexico (2012)

13. Ostfeld, A., Uber, J.G., Salomons, E., Berry, J.W., Hart, W.E., Phillips, C.A., Watson, J.P., Dorini, G., Jonkergouw, P., Kapelan, Z., di Pierro, F., Khu, S.T., Savic, D., Eliades, D., Polycarpou, M., Ghimire, S.R., Barkdoll, B.D., Gueli, R., Huang, J.J., McBean, E.A., James, W., Krause, A., Leskovec, J., Isovitsch, S., Xu, J., Guestrin, C., VanBriesen, J., Small, M., Fischbeck, P., Preis, A., Propato, M., Piller, O., Trachtman, G.B., Wu, Z.Y., Walski, T.: The battle of the water sensor networks (BWSN): A design challenge for engineers and algorithms. ASCE Journal of Water Resources Planning and Management 134(6), 556-568 (Nov/Dec 2008)

14. Schropp, D.: "smart building" technology for air safety monitoring: Sensor network design tool. Gases \& Instrumentation pp. 24-28 (Jul/Aug 2008)

15. Toregas, C., ReVelle, C.: Optimal location under time or distance constraints. Papers in Regional Science 28(1), 131-143 (Dec 1972)

16. U.S. Environmental Protection Agency (EPA): Exposure factors handbook: 2011 edition. National Center for Environmental Assessment, Washington, DC (Sep 2011), EPA/600/R-09/052F

17. Walton, G., Dols, W.: CONTAM 2.4 user guide and program documentation. National Institute of Standards and Technology, Gaithersburg, MD 20899-8633, 2.4c edn. (Oct 2005), nISTIR 7251

18. Wang, L., Dols, W., Chen, Q.: Using CFD capabilities of CONTAM 3.0 for simulating airflow and contaminant transport in and around buildings. HVAC\&R Research 16(6), 749-763 (Nov 2010)

19. Zhai, Z., Srebric, J., Chen, Q.: Application of CFD to predict and control chemical and biological agent dispersion in buildings. International Journal of Ventilation 2(3), 251-264 (Dec 2003) 